\title{
Alterstice
}

Revue internationale de la recherche interculturelle

International Journal of Intercultural Research

Revista International de la Investigacion Intercultural

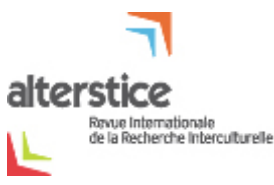

\section{Colloque de l'ARIC 2016, publications et congrès à venir}

\section{Elaine Costa-Fernandez}

Volume 6, numéro 1, 2016

URI : https://id.erudit.org/iderudit/1038273ar

DOI : https://doi.org/10.7202/1038273ar

Aller au sommaire du numéro

Éditeur(s)

Alterstice

ISSN

1923-919X (numérique)

Découvrir la revue

Citer ce document

Costa-Fernandez, E. (2016). Colloque de l'ARIC 2016, publications et congrès à venir. Alterstice, 6(1), 5-8. https://doi.org/10.7202/1038273ar d'utilisation que vous pouvez consulter en ligne. 


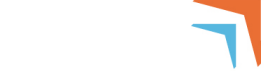 \\ alterstice
}

\section{Colloque de l'ARIC 2016, publications et congrès à venir}

\author{
Elaine Costa-Fernandez ${ }^{1}$
}

\section{Colloque de I'ARIC 2016}

Il faudrait faire en sorte que personne ne se sente exclu de la civilisation commune qui est en train de naître, que chacun puisse y retrouver sa langue identitaire, et certains symboles de sa culture propre, que chacun, là encore, puisse s'identifier, ne serait-ce qu'un peu, à ce qu'il voit émerger dans le monde qui l'entoure, au lieu de chercher refuge dans un passé idéalisé. Parallèlement, chacun devrait pouvoir inclure, dans ce qu'il estime être son identité, une composante nouvelle, appelée à prendre de plus en plus d'importance au cours du nouveau siècle, du nouveau millénaire : le sentiment d'appartenir aussi à l'aventure humaine. (Amin Maalouf, 1998, p. 187-188)

L'Association internationale pour la recherche interculturelle (ARIC), en partenariat avec le Programme de postgraduation en psychologie de l'Université fédérale de Pernambuco (UFPE) a organisé un colloque international du 26 au 29 avril 2016, dans un couvent du $16^{\mathrm{e}}$ siècle, sur le site historique d'Olinda-Pernambuco, au nord-est du Brésil. Avec l'organisation de cet événement, moment fort de rencontre scientifique, culturelle et humaine, I'ARIC a renforcé le dialogue avec des chercheurs d'institutions de "pays du Sud " - dialogue entamé à Florianópolis en 2006 -, élargissant son réseau de coopération scientifique avec des interlocuteurs des cinq continents. La thématique, de grande actualité, favorisait le croisement des regards sur les nouveaux défis pour la recherche scientifique et la pratique professionnelle : Mobilités, Réseaux et Interculturalité. L'ensemble des travaux a permis la confrontation et l'articulation entre différentes perspectives de recherche, en abordant des problématiques innovantes liées à l'interculturalité autour de trois axes.

- Mobilité : migration, déplacement, trajectoire

- Réseaux : réseaux numériques, réseaux professionnels, mouvements sociaux et politiques publiques

- Communication et interculturalité : nouvelles formes de communication et d'information, transmission culturelle et pratiques interculturelles contemporaines.

Cette manifestation scientifique a reçu l'appui financier d'organismes brésiliens comme la CAPES, la FACEPE, le Conseil fédéral de psychologie (CFP), I'Université catholique de Pernambuco (UNICAP), ainsi que d'organismes internationaux comme l'Agence universitaire pour la Francophonie (AUF), le Consulat général de France à Recife, I'Institut Français, I'Université fédérale Toulouse Midi-Pyrénées et l'Alliance française à Recife. Le comité d'organisation était présidé par Elaine Costa Fernandez, et le comité scientifique par Patrick Denoux ${ }^{1}$ et Aline Gohard ${ }^{2}$. Trente enseignants-chercheurs internationaux ont évalué plus de 300 résumés en français ou en portugais, les deux langues officielles du colloque, mais aussi en espagnol. Le nombre d'inscriptions a dépassé les attentes, avec 250 participants, dont les trois quarts venaient d’universités brésiliennes.

La programmation complète était composée de 3 mini-cours, 8 conférences plénières, 3 tables rondes, 9 symposia, 24 groupes de travail et 15 posters. Les trois mini-cours ont été consacrés à la médiation interculturelle, à la cyberpsychologie et à la méthodologie de la recherche interculturelle.

\footnotetext{
${ }^{1}$ Professeur en psychologie interculturelle, directeur de l'axe de psychologie interculturelle du Laboratoire cliniques psychopathologique et interculturelle (LCPI), Université de Toulouse Jean-Jaurès (France).

2 Professeure émérite en linguistique appliquée de l'Université de Fribourg (Suisse).
} 
Les trois tables rondes ont été animées

- pour l'axe 1 Nouvelles frontières physiques, symboliques, spatiales et développement durable par Julieta Maria Vasconcelos Leite, Caterine Regensi, Lucia Veras et Marcelo Pedroso, avec Reinaldo Fleuri comme médiateur,

- pour l'axe 2 Réseaux transnationaux et mobilité étudiante, par Michèle Vatz-Laaroussi, Liliana Kremer, Calos Canal, Chantal Doré et Jacques Caillouette, avec Lucienne Martins Borges comme médiatrice,

- pour l'axe 3 Communication et interculturalité par Véronique Donard, José Carlos Ribeiro et Julien Teyssier, avec Elaine Costa Fernandez comme médiatrice.

Les conférences plénières ont abordé des questions en rapport avec les thématiques du colloque. Nous voudrions souligner la participation de l'anthropologue Michel Agier, qui a donné une conférence sur la mobilité et les frontières, des philosophes Jacques Demorgon, sur la question des réseaux interculturels planétaires, et Charles Romain Mbele, sur la mondialisation en Afrique, ainsi que de psychologues venus parler des réseaux internationaux comme Odette Lescarret, à propos de l'Asie du Sud-Est, Claudio Bolzmann, au sujet des réseaux professionnels dans le domaine social et de la santé, et Mahmoud Benkhelifa, qui nous a partagé son expérience auprès du réseau international des méthodes projectives. Le psychanalyste Benoit Virole a présenté ses réflexions sur la cyberpsychologie et la diversité culturelle. Parallèlement, des activités culturelles ont été proposées, notamment trois concerts de musique classique et régionale et une présentation de frevo, danse locale reconnue Patrimoine immatériel de l'humanité par I'UNESCO.

Les divers travaux présentés ont abordé, dans une perspective transdisciplinaire, les défis que pose la globalisation à la démocratisation et à la diversité culturelle des réseaux de santé, des formations professionnelles et du monde universitaire. II ressort des débats que, si les questions posées sont transnationales, les réponses et défis doivent être abordés en tenant compte des impératifs locaux et de la pluralité des appartenances culturelles, dans une perspective interculturelle. La rencontre de plusieurs générations de chercheurs a été une occasion particulière de formation. Ourakoye Bembello et Audrey Lamothe, étudiantes en médiation interculturelle à l'Université de Sherbrooke, ont organisé un groupe de parole sur le concept d'interculturalité qui a été filmé et pourra être ensuite diffusé sur le site de l'ARIC pour un usage pédagogique. La dimension politique de l'interculturalité est apparue de façon évidente dans les études qui abordent la thématique des droits de la personne, l'inclusion numérique et les politiques publiques. Les questions associées à la mobilité urbaine, aux frontières et à la symbolique de l'espace ont aussi été abordées.

Plusieurs réseaux de recherche ont pu se rencontrer pour engager et élargir leur coopération, en particulier l'Association pour le développement de la psychologie en Asie du Sud-Est (ADEPASE), grâce à la présence de sa présidente, la professeure émérite en psychologie Odette Lescarret. Quelques groupes ont aussi consolidé des propositions de publications, des accords ou des projets communs, par exemple un groupe intéressé par des recherches sur "la radicalisation des jeunes et le terrorisme " sous la responsabilité du docteur Ghazi Chakroun (Université de Sfax). Des questions épistémologiques nouvelles dans le domaine de l'interculturalité ont aussi émergé.

En somme, ce colloque a mis en évidence la complexité des nouvelles thématiques de recherche dans la contemporanéité face à un contexte international marqué par l'hégémonie de la globalisation et les ruptures provoquées par le monde numérique tant au niveau des processus identitaires de subjectivation que dans les interactions sociales et les processus interculturels. Le phénomène de la mondialisation intervient dans la production du savoir et des technologies ainsi que dans le marché des communications, reconfigurant les processus d'interaction, de différenciation et d'identification entre différents groupes et mouvements socioculturels. Ces phénomènes pluriels font émerger de nouvelles questions épistémologiques, conceptuelles et méthodologiques, tant pour les professionnels que pour les chercheurs. À partir du constat que les modèles épistémologiques et politiques connus, y compris les modèles post-modernes pour certains, se montrent parfois inadaptés pour comprendre les mutations contemporaines, le colloque ARIC 2016 a tenté de dégager des pistes pour la démocratisation de nos sociétés, la réduction des inégalités sociales et la promotion de la diversité culturelle. 


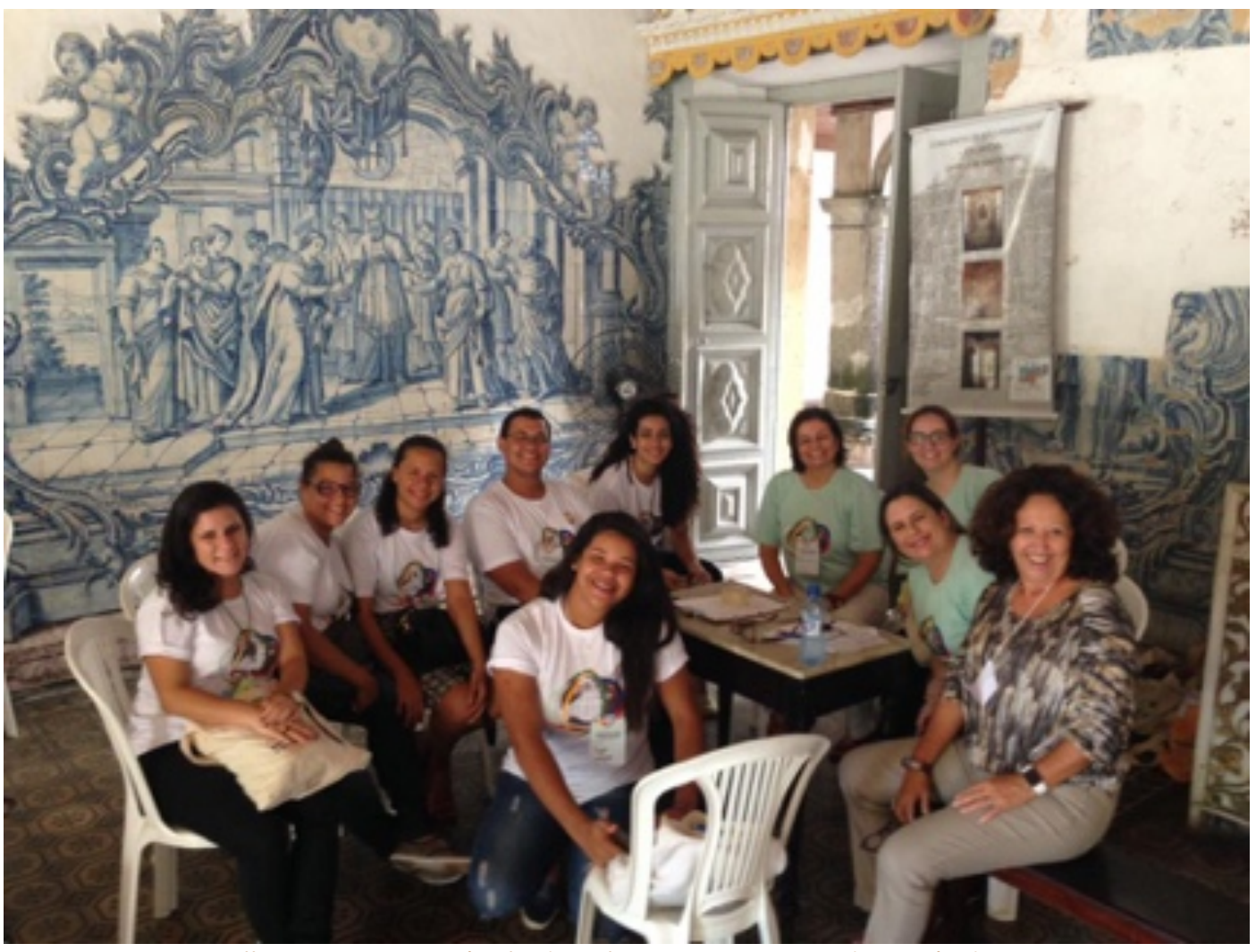

Colloque ARIC 2016 à Olinda : Elaine-Costa Fernandez, présidente, avec des membres du comité organisateur (en vert) et des bénévoles (en blanc).

\section{Publications}

Un ouvrage bilingue réunissant les textes complets de quelques participants du colloque ainsi que des contributions d'invités extérieurs, intitulé $O$ Psicólogo frente ao desafio tecnológico, novas identidades, novos campos, novas práticas, ainsi qu'un Cahier des résumés, ont été préparés par Elaine Costa Fernandez et Véronique Donard ${ }^{3}$.

o Psicólogo frente

ao desafio tecnológico
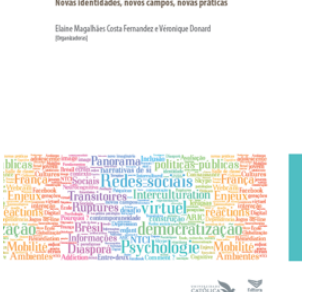

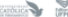

O Psicólogo frente ao desafio tecnológico,

novas identidades, novos campos, novas práticas

Deux ouvrages seront publiés avec des travaux complets, l'un dans la collection Espaces Interculturels chez L’Harmattan (Paris) et l'autre aux Éditions universitaires de l'Université fédérale de Pernambuco (Recife).

Deux numéros spéciaux seront aussi proposés aux comités scientifiques respectifs de deux revues en ligne : la revue Alterstice - Revue Internationale de la Recherche Interculturelle (Université Laval, Québec, Canada) et la revue Psychisme et Anthropos, Revue du Laboratoire d'anthropologie psychanalytique et de psychopathologie (Université d'Alger, Algérie).

\footnotetext{
${ }^{3}$ ISBN : 978-85-415-0752-3 pour l'ouvrage, ISBN : 978-85-415-0744-8 pour les résumés.
} 


\section{Congrès international EducA 2017}

L'ARIC est partenaire du congrès international EducA, qui aura lieu du 2 au 4 mars 2017 à Hammamet en Tunisie. Il portera sur la thématique Inégalités : quelles contributions des " éducations à... "?. Vous trouverez l'appel à communications et davantage de renseignements à ce sujet sur le site de l'ARIC (www.unifr.ch/ipg/aric/) ou sur le site du congrès (www.ecole-informatique.net/EducA2017).

\section{Congrès international ARIC 2017}

Le congrès de I'ARIC 2017 se déroulera à Antananarivo (Madagascar) du 23 au 26 mai 2017. II portera sur la thématique Nouveaux contextes et nouvelles pratiques interculturelles : Globalisation et circulation. Vous trouverez l'appel à communication sur le site de I'ARIC: http://www.unifr.ch/ipg/aric/. Un site internet est dédié au congrès avec des informations pratiques supplémentaires : http://congresaric2017.wixsite.com/creciantananarivo

\section{Rattachement de l'auteure}

${ }^{1}$ Université fédérale de Pernambuco (UFPE), Recife, Brésil

Elaine Costa-Fernandez Présidente de I'ARIC

\section{Correspondance}

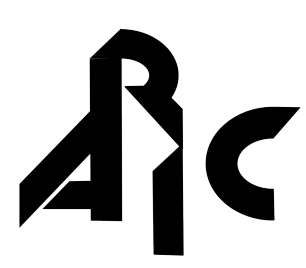

elainef@free.fr

\section{Pour citer cet article}

Costa-Fernandez, E. (2016). Colloque de I'ARIC 2016, publications et congrès à venir [Rubriqu'ARIC]. Alterstice, $6(1), 5-8$. 